HUNGARIAN AGRICULTURAL ENGINEERING

$N^{\circ} 37 / 2020$ 21-26

Published online: http://hae-journals.org/

HU ISSN 0864-7410 (Print) / HU ISSN 2415-9751(Online)

DOI: 10.17676/HAE.2020.37.21

Received: 20.10.2019 - Accepted: 15.01.2020
PERIODICAL OF THE COMITTEE OF

AGRICULTURAL AND BIOSYSTEM

ENGINEERING OF

THE HUNGARIAN ACADEMY OF SCIENCES

and

SZENT ISTVÁN UNIVERSITY

Faculty of Mechanical Engineering

\title{
DETECTION OF THE EFFICIENCY OF ENZYMATIC HYDROLYSIS AND FERMENTATION PROCESSES BY DIELECTRIC MEASUREMENT
}

\section{Author(s):}

S. Beszédes ${ }^{1}$, Z. Jákói ${ }^{1}$, B. Lemmer ${ }^{1,3}$, I. Czupy² and C. Hodúr ${ }^{1,3}$

\section{Affiliation:}

${ }^{1}$ Department of Process Engineering, University of Szeged, Faculty of Engineering, Moszkvai krt. 9., Szeged, H-6725, Hungary

${ }^{2}$ Institute of Forestry and Engineering and Environmental Technology, University of Sopron, Faculty of Forestry

Bajcsy Zs. street 4, Sopron, H-9400, Hungary

Institute of Environmental Science and Technology, University of Szeged

Tisza Lajos krt. 103, Szeged, H-6725, Hungary

\section{Email address:}

beszedes@mk.u-szeged.hu, czupy.imre@uni-sopron.hu, hodur@mk.u-szeged.hu

\begin{abstract}
In our work the efficiency of enzymatic degradation of cellulosic biomass was investigated by conventional analytical methods and dielectric measurements. Our results show, that the dielectric measurements proved to be efficient and rapid method to detect the chemical and physicochemical change of biomass during the enzymatic hydrolysis of cellulose. Because of molecular weight decreasing, and the increasing of the concentration of polar and ionic components, the polarization ability of the system has been changed, what manifested in the change of dielectric parameters. Furthermore, dielectric measurements were suitable to detect the efficiency of biomass pre-treatment, as well.
\end{abstract}

Keywords: fermentation, dielectric parameters, cellulose degradation, biomass

\section{Introduction}

Development of rapid methods to detect and quantify the efficiency of microbial and enzymatic degradation processes could be one of the key issues of R\&D activities in the field of biomass utilization. During the enzymatic hydrolysis, and in anaerobic digestion process the macromolecular components of biomass decompose to lower molecular weight components. In most of the biomass treatment processes (thermal, chemical and enzymatic methods) aim the preliminary hydrolysis of macromolecules and sludge matrix, as well. Dielectric measurements using microwave frequency ranges are suitable for sensing of biological materials and different kind of liquids because of their non-ionising nature, adequate penetration depth inside these samples, and good resolution due to their wavelength (Bircan et al., 2001). Study of dielectric parameters and dielectric behavior of materials helps to better understand the interaction between the materials and electromagnetic field, and has a good potential to detect some physical and physico-chemical change (Holtze et al., 2006).

Among the dielectric parameters the dielectric constant and dielectric loss factor are widely used to characterize the dielectric behavior of materials in radio frequency and microwave frequency ranges. Dielectric constant is the real part, dielectric loss factor is the imaginary part of the complex permittivity (Banik et al., 2003). The former measures the ability of a material to store the energy of electric field and the latter is a measure of the ability of a material to dissipate electric energy converting it into heat. Tangent loss is the tangent of the phase angles between the dipoles direction the irradiated material and the applied field (Metaxas and Meredith, 1993).

There can be found earlier study concerning of dielectric parameters of water and solution in microwave frequencies. Investigation of the dielectric properties of $\alpha$-D-glucose aqueous solution at microwave frequencies showed that the dielectric constant and dielectric loss factor decreased with concentration (Liao et al, 2001). This is due that the sugar molecules can form hydrogen bonds with the water dipoles, limiting 
the ability of water molecule for movement and decreasing its polarization capacity. But other study concluded that in higher concentration range of glucose solution (40-60 w\%), the change of concentration has any significant effect on the dielectric loss factor. Free water molecules form hydrogen bonds with hydroxyl groups of carbohydrates, suppressing the internal hydrogen bonds in glycosidic bridge. This phenomena contributes to achieve enhanced mobility of glycosidic linkage and worse mobility of hydroxymethyl group. The change of hydrogen bond characteristics can be considered as an explanation of the behavior of relaxation processes in the sample saturated with water (Kaminski et al., 2010). In cellulose contented materials and suspensions the presence of absorbed water significantly changes dielectric properties of polysaccharides (Shinouda and Moteleb, 2005).

At a given concentration, temperature increasing led to increased dielectric constant. It was also verified, that the dielectric constant at lower temperature is more influenced by the glucose concentration than that of observed at higher temperature range (Hochtl et al, 2000). In two component system the dielectric parameters can be estimated based on the observation that the dielectric constant and concentration of carbohydrates has a near linear correlation. It should be noticed, that these simplification presumed that there is no interaction between the two components. Electric conductivity of ionic solutions increases with temperature, because of decreased viscosity and enhanced mobility of the ions. Beside inconsistencies in literature based data, further and deeper investigations are needed to discover matrix effects in suspensions and multicomponent solutions. In fermentation broth produced in anaerobic digestion or enzymatic hydrolysis of lignocellulosic biomass amino acids are presented. If temperature reach a critical value Maillard-type reactions can be occurred between reducing sugars (such as glucose, fructose, xylose, galactose) and free amino acids. Reactive carbonyl group of reducing sugars with nucleophile amino groups of amino acids form heavily characterizable, but higher molecular weight compounds. Maillard-type reactions are accelerated in alkaline condition (Liao et al., 2003). These chemical changes have effect on the complex polarization ability of multicomponent system.

In aqueous solution, especially in supersaturated systems, the effects of concentration increasing on dielectric parameters are influenced by the change of ratio of free to bound water (Fuchs and Kaatze, 2001). Free water molecules have dielectric properties very similar to those that of obtained for liquid water, but the dielectric behavior of bounded water is near to properties that obtained for ice. But it can be noticed that the temperature increment has stronger effect on dielectric parameters of bounded water than that of detectable for free water, because at high temperature the mobility of bounded water increase. In ethanol-water system the ethanol molecule behave as a solute, it forms hydrogen bounds in the hydroxyl groups, decreasing the free rotation ability of the water molecules (Bohigas and Tejada, 2010). These effects led to decreased capability to store and dissipate the energy carried by electromagnetic waves, which manifested in reduced dielectric constant and dielectric loss factor (Sato et al., 2004; Olmi et al., 2007).

Dielectric parameters decidedly provide information about the interaction between electromagnetic field and materials, and has good potential to detect chemical and structural changes, but, however, the change of dielectric constant and loss factor has also effect on the heat generation ability and overall thermal efficiency of microwave irradiation (Leonelli and Mason, 2010). High scale absorption of electromagnetic waves occurs for materials with high dielectric permittivity or high magnetic permeability (Khan et al., 2014). Water molecule, and the high water contented systems and materials have a relatively high dielectric loss factor. Therefore the penetration depth of microwave irradiation in these systems are relatively short. In the frequency range of $900 \mathrm{MHz}$ to $2450 \mathrm{MHz}$ the temperature profile of materials resulted by the exposure to electromagnetic field is dependent on both the dielectric constant and the loss factor, not just on dielectric constants as mentioned often in the literature.

Microwave irradiation, as electromagnetic field, has a specific effect to polarize the side chains of macromolecules. Therefore a change in dipole orientation of polar components can be occurred. Depending of the frequency the water molecules with dipolar characteristics is moving in time with the continuously varying polarity electromagnetic field. Intensity of this movement is influenced by the strength of hydrogen bound formed in materials (Brodie et al., 2014). It is verified for wastewater and sludge matrix that due to hydrothermal and chemical treatment the high molecular weight macromolecular components are partially hydrolyzed, and extracellular polymeric substances and cell walls are disintegrated what increase the concentration of ionic and low molecular weight polar components in liquid phase. With the increment of 
mobility and concentration of components with higher polarization ability in electromagnetic filed, dielectric parameters are assumed to changed.

In our research work we focused on the applicability of dielectric measurements to detect the degree of biodegradation process for wastewater sludge, and the degree of cellulose degradation in controlled enzymatic hydrolysis process, respectively.

\section{Materials and methods}

In our work the biodegradation efficiency of microwave pre-treated meat industry wastewater sludge was investigated i) under aerobic condition by using of respirometric biochemical oxygen demand (BOD) measurements $\left(20^{\circ} \mathrm{C}\right.$ for 5 days $)$, and ii) under anaerobic condition by using of continuously stirred batch mesophilic $\left(37^{\circ} \mathrm{C}\right)$ anaerobic digestion tests. Enzymatic degradation degree of cellulosic biomass (corn cob) was tested by Cellic Ctec 2 enzyme (dosage of $30 \mathrm{U} / \mathrm{g}$ ), reducing sugar (RS) content was measured by spectrophotometric reducing sugar assay (DNSA method).

Beside the commonly used analytical parameters the dielectric parameters (dielectric constant, dielectric loss factor, loss tangent, reflection coefficient) were determined, as well. For dielectric measurements a DAK3.5 (SPEAG, Switzerland) dielectric sensor connected to a vector network analyser (ZVL3, Rohde \& Schwarz, Germany) was applied in a frequency range of $200 \mathrm{MHz}$ to 2,400 MHz. Average values of dielectric parameters was calculated from the data obtained 60 measurements. Samples are thermostated at $25^{\circ} \mathrm{C}$ during dielectric measurements.

\section{Results and discussion}

Continuously flow microwave (MW) pre-treatments were carried out with different power intensity (418W and $700 \mathrm{~W}$ ) and varied volumetric flow rate (in the range of 28.5-48.5 Lh-1) for meat processing wastewater sludge. Efficiency of MW pre-treatment was characterized by the ration of biochemical oxygen demand (BOD) to chemical oxygen demand (COD) parameter which corresponds to the ratio of biodegradable (under aerobic condition) to total organic matters. Due to the MW pre- treatments, the concentration of soluble and biodegradable organic matters increased, therefore the BOD/COD improved, as well. Dielectric constant sludge was measured after MW pre-treatment (samples were cooled to $25^{\circ} \mathrm{C}$ ). Figure 1 show the correlation between the aerobic biodegradability and dielectric constant for sludge treated by $418 \mathrm{~W}$ and $700 \mathrm{~W}$ power intensity MW irradiation.

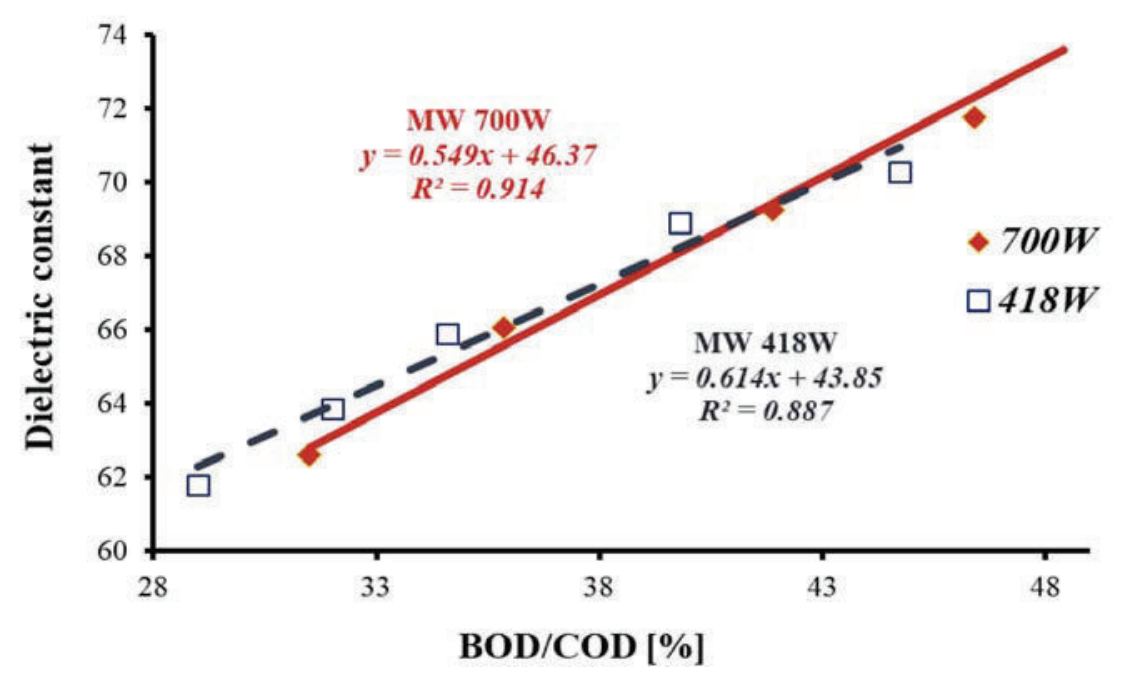

Figure 1. Correlation between dielectric constant (measured at $2400 \mathrm{MHz}$ ) and aerobic biodegradability (given by the ratio of biochemical oxygen demand to chemical oxygen demand: BOD/COD) for microwave pre-treated meat processing wastewater sludge (power intensity of $418 \mathrm{~W}$ and $700 \mathrm{~W}$ ) 
Our results verified, that due to the intensive disintegration effect of MW irradiation the free water content, the migrable ionic components and soluble organic matter content increased what manifested in higher ability for polarization in electromagnetic field. Thermal effect of MW resulted in decomposition of macromolecules, disruption of cell walls, liberation of polar and ionic compounds from intracellular space into sludge liquor, and produce of lower molecular weight components (Dogan and Sanin, 2009). These effects led to higher biodegradability and increment of dielectric constant, respectively. Independently from the applied power intensity for MW treatment a strong linear correlation was observed between the BOD/COD and dielectric constant.

To further examination of the efficiency of MW treatment on sludge mesophilic anaerobic digestion (AD) tests were carried out to determine the biodegradability under anaerobic condition. For MW pre-treatments prior to $\mathrm{AD}$ tests a continuously flow MW equipment was used with varying the volumetric flow rate of sludge pumping through the MW reactor. Biodegradability under anaerobic condition was characterized by the biogas production, given by the percentage increment of biogas volume related to the control (non-treated) sample (Figure 2).

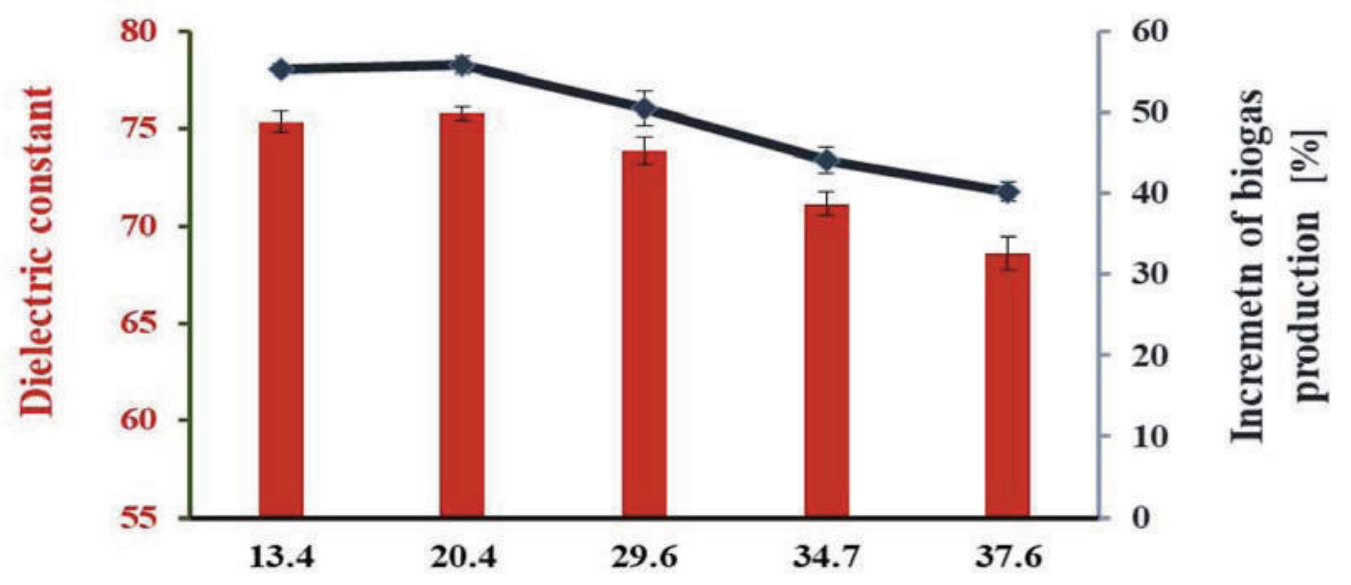

Volumetric flow rate $\left[\mathrm{L} \mathrm{h}^{-1}\right]$

Figure 2. Change of dielectric constant $(2400 \mathrm{MHz})$ and increment of biogas production of sludge (related to untreated sample, produced at $37^{\circ}$ ) as a function of volumetric flow rate of continuously flow microwave pre-treatment (at power level of $700 \mathrm{~W}$ )

Results of AD tests show, that MW pre-treatments were suitable to increase the biogas yield from meat processing sludge. Biogas production increased with decreasing of volumetric flow rate of sludge in MW process (which means prolonged residence time in MW reactor). Dielectric constant of MW pre-treated sludge samples was measured after cooling to temperature of $\mathrm{AD}$ test $\left(37^{\circ}\right)$ and before anaerobic inoculum dosage. It has been revealed, that the change of biogas production versus volumetric flow rate show similar tendency that of obtained for the dielectric constant measured at the frequency of $2400 \mathrm{MHz}$.

Applicability of dielectric measurements for detection of biodegradation degree was tested during enzymatic hydrolysis of lignocellulosic biomass (with corn cob residues using high cellulose activity enzyme blend).

Efficiency of enzymatic degradation process was characterized by the concentration of reducing sugars (RS) produced from the hydrolysis of cellulose. Different from the dielectric behavior of sludge, in cellulose hydrolysis process the dielectric loss factor has been proved to be suitable to detect the change of biodegradation degree. In MW processed sludge the dielectric constant at measuring frequency of $2450 \mathrm{MHz}$ was sufficiently sensitive to measure the change of aerobic and anaerobic biodegradability, respectively. Dielectric loss factor decreased with increased sugar concentration, which tendency was observed concluded in preliminary studies (Liao et al, 2001; Shinouda and Moteleb, 2005) 


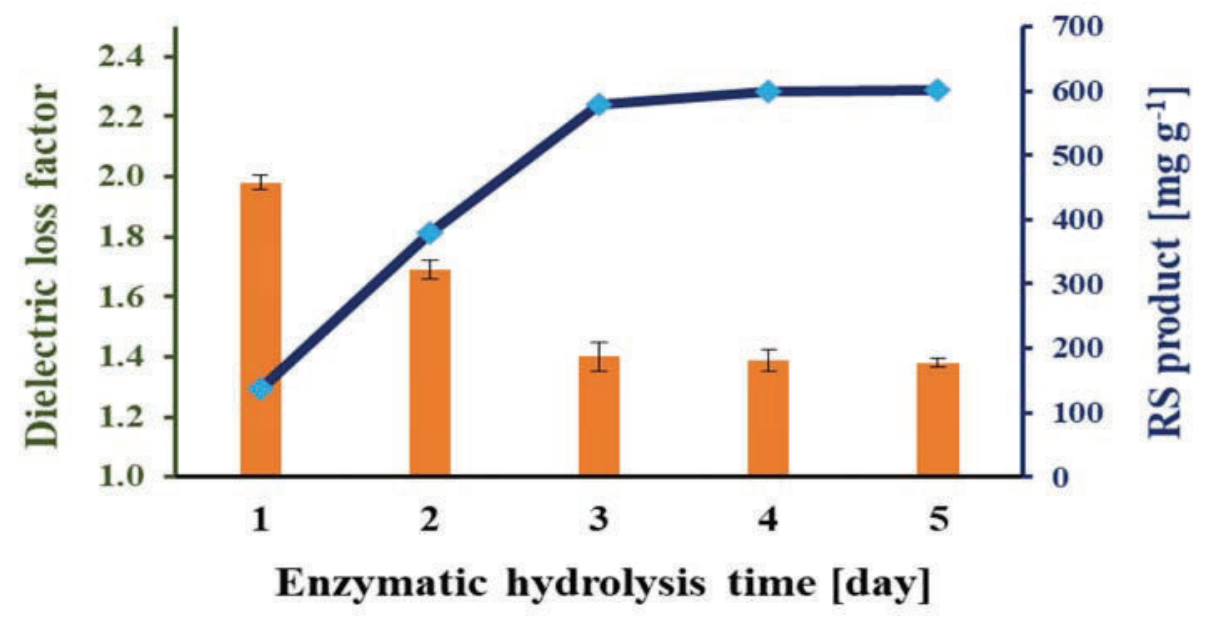

Figure 3. Change of dielectric loss factor (measured at frequency of $400 \mathrm{MHz}$ ) and reducing sugar concentration during enzymatic hydrolysis of lignocellulosic biomass $\left(40^{\circ} \mathrm{C}\right)$

In high carbohydrate contented biomass suspension had to decrease the measuring frequency of dielectric loss factor to $400 \mathrm{MHz}$ to find correlation between dielectric behavior and the change of RS concentration. In this frequency the increment of RS concentration caused the decrease of dielectric loss factor. The tendency of the change of loss factor and RS concentration show good inverse correlation (Figure 3).

\section{Conclusion}

In our research the applicability of dielectric measurement for detection of biodegradation processes was investigated. Our results verified that both the change of biodegradability of meat processing sludge under aerobic and anaerobic condition, and the change of the degree of enzymatic hydrolysis of lignocellulosic biomass can be detectable by dielectric measurements.

In sludge sample matrix the ratio of biodegradable to total organic matter content (given by BOD/COD ratio) and the improvement of biogas production show good correlation with dielectric constant at the frequency of $2450 \mathrm{MHz}$. Degree of enzymatic cellulose degradation (characterized by reducing sugars produced from cellulose hydrolysis) correlate with dielectric loss factor measured at the frequency of $400 \mathrm{MHz}$. Our preliminary results enable to develop rapid, non-destructive method for the control of enzymatic and microbial biodegradation processes, and, however, contibute to define in-line and/or on-line efficiency estimation algorithm and methods for different pre-treatments.

\section{Acknowledgements}

The support of European Union and Hungarian State in the framework of the project entitled 'Thematic Network for the Sustainable Use of Resources - RING207' (program code: EFOP-3.6.2-16-2017-00010) is gratefully appreciated. The authors are grateful for the financial support provided by the NRDI (NKFI) under project number K115691.

\section{References}

[1] Banik, S., Bandyopadhyay, S., Ganguly, S. (2003), Bioeffects of microwave-a brief review. Bioresource Technology, Vol. 87., pp. 155-159.

[2] Bircan, C., Barringer, S.A., Mangino, M. (2001), Use of dielectric properties to detect whey protein denaturation. Journal of Microwave Power and Electromagnetic Energy, Vol. 36, pp. 179-186.

[3] Bohigas, X., Tejada, J. (2010), Dielectric characterization of alcoholic beverages and solutions of ethanol in water under microwave radiation in the $1-20 \mathrm{GHz}$ range. Food Research International, Vol. 43, pp. 1607-1613 
[4] Brodie, G., Destefani, R., Schneider, P.A., Airey, L., Jacob, M.V. (2014), Dielectric properties of sewage biosolids measurement and modeling. Journal of Microwave Power and Electromagnetic Energy, Vol. 48, pp. $147-157$.

[5] Dogan, F.D., Sanin D. (2009), Alkaline solubilization and microwave irradiation as a combined sludge disintegration and minimization method. Water Research, Vol. 43(8), pp. 2139-2148.

[6] Fuchs, K., Kaatze, U. (2001), Molecular dynamics of carbohydrate aqueous solutions: dielectric relaxation as a function of glucose and fructose concentration. The Journal of Physical Chemistry B, Vol. 105, pp. 2036-2042.

[7] Hochtl, P., Boresch, S., \& Steinhauser, O. (2000), Dielectric properties of glucose and maltose solutions. Journal of Chemical Physics, Vol. 112, pp. 9810-9821.

[8] Holtze, C., Sivaramakrisham, R., Antionetti, M., Tsuwi, J., Kremer, F., Kramer KD (2006), The microwave absorption of emulsions containing aqueous micro and nanodroplets: a means to otpimize microwave heating. Journal of Colloid and Interface Science, Vol. 302, pp. 651-657.

[9] Kaminski K, Wlodarczyk P, Adrjanowicz K. (2010), Origin of the commonly observed secondary relaxation process in saccharides. Journal of Chemical Physics B, Vol. 114, pp.11272-11281.

[10] Khan SUD, Arora M, Wahab MA, Saini P (2014), Permittivity and electromagnetic interference shielding investigations of activated charcoal loaded acrylic coating compositions. Journal of Polymers, 2014, pp. 1-7.

[11] Liao, X., Raghavan G.S.V., Dai, J., Yaylayan V.A. (2003), Dielectric properties of a-d-glucose aqueous solutions at $2450 \mathrm{MHz}$. Food Research International, Vol. 36, pp. 485-490.

[12] Leonelli C., Mason T.J. (2010), Microwave and ultrasonic processing: Now a realistic option for industry. Chemical Engeneering and Processing, Vol. 49, pp. 885-900.

[13] Metaxas, A.C., Meredith, R.J., 1993. Industrial microwave heating. IEE Power Engineering Series 4. Peter Peregrinus Ltd., United Kingdom, London.

[14] Olmi, R., Meriakri, V.V., Ignesti, A., Priori, S., Riminesi, C. (2007), Dielectric spectroscopy of sugar and ethanol solutions in water for monitoring alcoholic fermentation processes. Journal of Microwave Power Electromagnetic Energy, Vol. 41(3), pp. 37-49.

[15] Sato, T., Buchner, R. (2004), Dielectric relaxation processes in ethanol/water mixtures. Journal of Physical Chemistry, Vol. 108, pp. 5007-5015

[16] Shinouda HG, Moteleb MMA (2005), Dielectric spectroscopy and relaxation phenomena of moistened and dry polysaccharides. Journal of Applied Polymer Science, Vol. 98, pp.571-582

[17] Velázquez-Varela, J., Castro-Giráldez, MFito ., P.J. (2013), Control of the brewing process by using microwaves dielectric Spectroscopy. Journal of Food Engineering, Vol. 119, pp. 633-639 